\title{
El Impacto de la Aproximación Fondos de Conocimiento desde la Visión de las Docentes y las Familias. Un Estudio Cualitativo
}

\section{The Impact of the Funds of Knowledge Approach from Teachers' and Families' perceptions. A Qualitative Study}

\author{
Mireia Machancoses * \\ Universitat de Girona, España
}

\section{DESCRIPTORES:}

Fondos de conocimiento

Relaciones familia-

escuela

Perspectiva del déficit

Familias migrantes

\section{RESUMEN:}

Un gran número de investigaciones han demostrado los beneficios de unas buenas relaciones familia-escuela en el desarrollo y aprendizaje del alumnado, especialmente en situaciones de discontinuidad entre la familia y la escuela. En este sentido, el programa fondos de conocimiento se propone como una aproximación inclusiva para facilitar los procesos de contextualización educativa y generar relaciones de confianza mutua entre docentes y familias. En el presente estudio se ha aplicado el programa en un aula de educación infantil, con niños y niñas de 3 años de edad, en un centro público con un elevado índice de alumnado con familias procedentes del extranjero, analizando la visión de las docentes y, por primera vez en la literatura disponible, de las familias participantes. Los instrumentos utilizados fueron cuestionarios administrados antes y después de la participación en el proyecto, para las docentes participantes, así como entrevistas a las familias tras las visitas etnográficas realizadas por las docentes. Los resultados muestran la creación de unos vínculos más sólidos entre las docentes y las familias participantes. En particular, las familias destacan una mayor confianza hacia las docentes, una mayor participación en la escuela, y especialmente destacan la valorización y reconocimiento de sus conocimientos como aspecto positivo.

\section{KEYWORDS: \\ Funds of knowledge \\ Family-school \\ Relationships \\ Deficit perspective \\ Migrant families}

\begin{abstract}
:
A large number of studies have demonstrated the benefits of a positive home-school relationships for the development and learning processes of children. In particular, these benefits are more salient in home-school discontinuities situations characterized by a high sociocultural diversity. In this context, the funds of knowledge approach arise as an inclusive approach to facilitate educational contextualization processes and to generate relationships of mutual trust between teachers and families. In the present study, the program has been implemented in a classroom with 3-year-old children, from a public school with a high rate of students with families from abroad, analyzing the perception of the teachers and, for the first time in the available literature, of the participating families. The instruments used for the research were questionnaires, administrated before and after the funds of knowledge approach design and implementation, given to teachers and interviews to family members after the ethnographic visits carried out by teachers. The results show the establishment of stronger relationships between teachers and participant families. In particular, families highlight a greater trust towards teachers, a higher participation in the school and in particular they emphasize the appreciation and recognition of their skills, knowledge, and practices as a positive aspect.
\end{abstract}

CÓMO CITAR:

Machancoses, M. (2021). El impacto de la aproximación fondos de conocimiento desde la visión de las docentes y las familias. Un estudio cualitativo. Revista Internacional de Educación para la Justicia Social, 10(1), 55-68.

https://doi.org/10.15366/riejs2021.10.1.004

*Contacto: mireiareal@gmail.com

ISSN: 2254-3139

revistas.uam.es/riejs 


\section{Revisión de la literatura}

Existe un considerable corpus empírico que demuestra los beneficios de la implicación parental y unas buenas relaciones familia-escuela, entendidas como el conjunto de interacciones entre docentes y padres/madres con el objetivo de conectar ambos contextos de socialización, teniendo el desarrollo y aprendizaje del alumno/a como objetivo principal y compartido (Epstein, 2018; Rodríguez-Ruiz et al., 2019).

En este sentido, distintas propuestas de intervención se han llevado a cabo con el objetivo de mejorar las relaciones familia-escuela. Especialmente nos centramos en este artículo en la aproximación conocida como "fondos de conocimiento" (Moll, 2019). En particular, se desconocen las contribuciones específicas de dicho programa de investigación-acción en la mejora de dichas relaciones según la perspectiva de las familias participantes. Para subsanar y contribuir a la literatura existente, la pregunta de investigación fundamental que subyace en este artículo fue: ¿qué impacto tiene el programa en la mejora de las relaciones familia-escuela según los docentes y familias participantes?

Dividimos esta introducción en tres subsecciones. En primer lugar, justificamos los beneficios de unas buenas relaciones familia-escuela según la literatura disponible. En segundo lugar, contextualizamos brevemente la aproximación de los fondos de conocimiento como enfoque específico de investigación e intervención para propiciar unas mejores relaciones entre docentes y familias. Finalmente, describimos los objetivos de la investigación.

\subsection{La importancia de unas buenas relaciones familia-escuela}

Las revisiones de la literatura efectuadas por Blasco (2018), Henderson y Mapp (2002) o Semke y Sheridan (2012) muestran el impacto positivo que tienen unas fluidas y positivas relaciones familia-escuela en el rendimiento académico de los/as estudiantes, así como en general en el aprendizaje y desarrollo de los mismos.

En esta línea, el análisis de García-Bacete (2003) establece que dichos beneficios repercuten en tres niveles: 1) En los estudiantes: mejora de las notas, actitudes más favorables en relación a las tareas escolares, conductas más adaptativas, una mayor autoestima, realización de los deberes, participación en las actividades del aula, menor escolarización en programas de educación especial, menor tasa de abandono y absentismo; 2) En los docentes: mejores habilidades interpersonales y de enseñanza, mayor satisfacción profesional y mayor compromiso con la instrucción; 3) En las familias: aumento del sentido de autoeficacia, incremento de la comprensión de los programas escolares, valoración del rol que tienen en relación a la educación de sus hijos e hijas, mayor motivación para continuar su propia educación, mejora de la comunicación con sus hijos/as en general y sobre las tareas escolares en particular, desarrollo de habilidades positivas hacia la paternidad.

Asimismo, estos beneficios parecen ser especialmente sensibles en aquellos casos caracterizados por una mayor discontinuidad entre los códigos y prácticas escolares y familiares, por ejemplo, en situaciones de desigualdad económica o diferencia cultural, así como de estigmatización basadas en la existencia de prejuicios y estereotipos (Collet et al., 2014; Crosnoe, 2012; Esteban-Guitart y Bastiani, 2010; Poveda, 2001; Sharma, 2018). En estos casos puede existir un desconocimiento del sistema educativo, prejuicios y estereotipos e incluso conflictos culturales dada la dificultad para entender matices o el funcionamiento, códigos y prácticas del centro escolar (Esteban-Guitart y Vila, 2013a).

En este sentido, se ha identificado un discurso dominante que apunta a la explicación del rendimiento escolar a partir del desconocimiento y supuesta falta de implicación de las familias de grupos culturales minorizados (Besalú y Vila, 2007). Lo que la literatura recoge bajo la "perspectiva del déficit en educación" (Valencia, 2010), atribuyendo a las familias su propio fracaso y afirmando que son éstas las que deberían mejorar; conocer e implicarse más y mejor en la escolaridad de sus hijos/ as, sin tener en cuenta los elementos estructurales e institucionales subyacentes (Ainscow, 2012; Essomba, 2003; Esteban-Guitart y Vila, 2013b; Vila y Esteban-Guitart, 2017). 
De hecho, el desconocimiento mutuo en situaciones de discontinuidad familia-escuela (Poveda, 2001) puede conducir a prejuicios en ambos sentidos que dificulten, precisamente, unas buenas relaciones, así como el establecimiento de alianzas basadas en la confianza mutua (Llopart et al., 2017), lo que supone procesos de conocimiento y reconocimiento que obligan, según la terminología de González y Moll (2002), a “cruzar el Puente” (p. 623) entre el hogar y la escuela.

En la terminología del modelo sistémico-ecológico de Bronfenbrenner (1979), se trata de facilitar la creación de "mesosistemas", es decir, promover el aprendizjae y desarrollo de las criaturas a partir de la articulación de contextos de vida y socialización, como son la escuela y la familia. Estas conexiones pueden tomar la forma de actividades compartidas, comunicación en los dos sentidos (intercambio de informaciones), la aparición de confianza mutua, una orientación positiva, el consenso de metas, así como un equilibrio de poderes hacia relaciones más horizontales (Gifre y Esteban-Guitart, 2012).

\subsection{El impacto de la aproximación de los fondos de conocimiento}

El enfoque Fondos de conocimiento surgió hace más de tres décadas en la Universidad de Arizona (USA) para desafiar la perspectiva del déficit, anteriormente mencionada (González et al., 2005; Moll et al., 1992). El propósito fundamental consiste en mejorar el rendimiento escolar a partir de procesos de contextualización curricular, y mediante la mejora de las relaciones entre docentes y familias. En la actualidad, a pesar de que se sigue aplicando principalmente en los Estados Unidos de América, se ha desarrollado también en otros contextos y países como España, Nueva Zelanda o Australia (Esteban-Guitart, 2021; Esteban-Guitart y Moll, 2014a, 2014b; Hogg, 2011; Llopart y Esteban-Guitart, 2018).

Uno de los aspectos más destacados del programa son las visitas que los docentes realizan a los hogares de algunos de sus alumnos/as para identificar los fondos de conocimiento de los que disponen las familias. "Las familias son contextos de vida y escenarios de enseñanza y aprendizaje, al igual que el contexto escolar, de modo que se trata de conocer sus fortalezas para poderlas visibilizar y utilizar pedagógicamente" (Esteban-Guitart y Saubich, 2013, p. 111). Después de estas visitas, se crean unidades didácticas que vinculan los fondos de conocimiento identificados con los objetivos de aprendizaje. Esto facilita que los aprendizajes del alumnado sean significativos, ya que conectan las experiencias y los conocimientos previos con lo que se hace en la escuela. En este sentido, la finalidad es que estos conocimientos previos les ayuden a conectar con los nuevos aprendizajes y contenidos de la institución escolar (Esteban-Guitart y Saubich, 2013, p. 191). El objetivo no es que se haga en la escuela lo mismo que en el hogar, sino que las fuentes de conocimiento de las que disponen apoyen y expandan sus aprendizajes (Esteban y Vila, 2013b).

En particular, a nivel metodológico, se han recogida evidencias del impacto del programa a partir de narrativas que relatan las experiencias por parte de los propios docentes durante las visitas (Tenery, 2005); a partir del análisis de las experiencias de los docentes antes, durante y después de las visitas (Esteban-Guitart y Vila, 2013b; Whyte y Karabon, 2016); en la información extraída de diarios reflexivos escritos por los propios docentes (Lin y Bates, 2010); así como también con cuestionarios y entrevistas en profundidad (Lin y Bates, 2010; Meyer y Mann, 2006). La conclusión principal de estos trabajos es que los docentes dicen crear relaciones mucho más cercanas y positivas con las familias, mejorando la comprensión, empatía y comunicación con ellas, como consecuencia de las visitas que han permitido conocer mejor las condiciones históricas y culturales del hogar, así como crear relaciones más informales de intercambio. En particular, se valora positivamente el mayor conocimiento empírico del alumnado y las familias que permite, por otra parte, modificar prejuicios y estereotipos.

No obstante, no existen estudios que analicen el impacto que tiene el programa, en concreto las "visitas etnográficas" (Llopart et al., 2017) realizadas por los docentes en los hogares de algunos de sus estudiantes, sobre las percepciones, creencias y actitudes de las familias en relación a la escuela, los docentes y las relaciones entre ambos contextos y realidades, pues los estudios disponibles se han focalizado en el análisis de las percepciones y creencias de los docentes. 
En este sentido, se pretende contribuir a la literatura existente acerca de las relaciones familia-escuela a partir de la aproximación de los fondos de conocimiento analizando, por primera vez en la literatura disponible al respecto, el punto de vista de las familias en relación a la escuela y el impacto del programa sobre las familias visitadas según su propia experiencia. También se exploran las visiones del equipo docente con la finalidad de contrastar las vivencias de ambos agentes sociales y educativos.

\section{Objetivos de la investigación}

El objetivo principal que ha guiado la presente investigación ha sido, mediante la implementación del programa fondos de conocimiento en un contexto escolar con alto índice de alumnado inmigrante, identificar los factores que inciden en la mejora de las relaciones familia-escuela tanto desde la visión de los docentes como de las familias implicadas. Dentro del objetivo principal, se han abordado distintos objetivos específicos. En concreto presentamos en este artículo algunos resultados vinculados a los siguientes objetivos y propósitos de la investigación:

- Definir unas óptimas relaciones familia-escuela según las docentes y las familias participantes en el programa;

- Describir los beneficios que se derivan de unas óptimas relaciones entre la familia y la escuela según las docentes y las familias participantes;

- Identificar la contribución del programa educativo fondos de conocimiento en la mejora de las relaciones familia-escuela según las docentes y las familias participantes.

\section{Método}

Dentro del análisis cualitativo, este estudio se lleva a cabo en el marco de la "teoría fundamentada" (Glaser, y Strauss, 1967) basada en la construcción de códigos y categorías a partir del análisis del corpus textual o material empírico. Este procedimiento parte del análisis inductivo de los datos cualitativos para construir una teoría determinada sobre el objeto de estudio, siendo el proceso de codificación teórica de especial relevancia en el procedimiento. "Su principal diferencia respecto a otras metodologías cualitativas es que pone un gran interés en el desarrollo de una teoría sustentada en los datos procedentes del trabajo de campo" (Hernández, 2014, p. 192).

En concreto, se crearon distintas categorías asociadas a las distintas preguntas de investigación, a saber: caracterizar unas óptimas relaciones familia-escuela, describir los beneficios que se derivan de ellas, así como identificar la contribución que el programa educativo fondos de conocimiento potencialmente tiene en la mejora de las relaciones familia-escuela. Se lleva a cabo el análisis mediante la comparación entre las percepciones y voces de las docentes y de las familias participantes en el estudio.

\section{Contexto de la investigación}

La implementación de la aproximación "fondos de conocimiento" en la escuela fue propuesta y facilitada por el equipo de investigación tras contar con la colaboración y voluntad del equipo directivo del centro, y tras compartir con el claustro, y las docentes finalmente participantes, la naturaleza del estudio. En este sentido, se obtuvo consentimiento informado de todas las participantes en el proyecto, tanto docentes como familias. El estudio se realizó en un centro educativo de titularidad pública ubicado en la ciudad de Girona y caracterizado por un alto índice de alumnado con familias de origen extranjero. En un primer momento, como se ha dicho anteriormente, se presentaron los objetivos y características de la aproximación fondos de conocimiento a la dirección y el claustro del centro educativo. Tras la valoración de dicha aproximación, el centro mostró interés en aplicarlo y para ello se creó un grupo de estudio formado por las docentes e investigadoras de la universidad, a través del cual se materializaron las diferentes fases del programa educativo: una primera de formación, la preparación del trabajo de campo, la identificación de los fondos de conocimiento y su extrapolación a actividades educativas en el aula (Esteban-Guitart et al., 2018). 
En todas las fases del proyecto, las investigadoras adoptaron un rol de acompañamiento y colaboración con las participantes, tanto docentes como familias. Los compromisos que se adoptaron estuvieron vinculados a facilitar la contextualización de la práctica docente a partir de la aproximación de los fondos de conocimiento en lo referente al andamiaje teórico y metodológico para mejorar las relaciones con las familias, así como los aprendizajes del alumnado. En una última sesión del grupo de estudio-grupo de trabajo formado por docentes e investigadoras, se valoró la experiencia mediante un análisis de las fortalezas, debilidades y aspectos de mejora de la implementación de la perspectiva de los fondos de conocimiento llevado a cabo.

\section{Participantes}

En la implementación de la aproximación "fondos de conocimiento" han participado un total de 6 docentes pertenecientes al ciclo de Educación Infantil. Todas ellas han sido mujeres, por lo que se utiliza el género femenino para referirse a las docentes participantes en el estudio. Los años de experiencia docente varían desde los ocho meses hasta los nueve, diez, doce, dieciséis y veintiséis años. La escuela fue la que eligió qué docentes participarían en el estudio, todas ellas compañeras de ciclo y con un vínculo educativo con el alumnado y las familias participantes.

Cada docente escogió una familia con hijos e hijas escolarizados en su aula, segundo ciclo de educación infantil (niños y niñas de 3 años de edad), de modo que participaron en total seis familias en el estudio. El motivo de aplicarlo en dicho contexto educativo, por parte de la escuela, fue poder trabajar con estas familias a lo largo de toda la escolarización de sus hijos/as, mejorando las relaciones familia-escuela des del momento inicial de la escolarización en el centro. De modo que se consideró que la aproximación de los fondos de conocimiento podría ser adecuada para obtener un mejor conocimiento de las familias visitadas, así como del alumnado recién incorporado en el centro.

En particular, los tres criterios principales que siguieron las docentes para escoger a las familias fueron: 1) familias en las que se observaba una discontinuidad en la relación familia-escuela en forma de desconocimiento o poca relación; 2) familias que tuvieran un conocimiento de la lengua de acogida que permitiera realizar las entrevistas y, finamente, 3) se priorizó que éstas representaran culturas diversas, reflejo de la heterogeneidad presente en el centro educativo, en el barrio y en la zona de la ciudad. Con la aplicación de dichos criterios, las docentes seleccionaron una familia procedente de Honduras, una de la India, dos de Marruecos y dos de ellas procedentes de Gambia. Cabe destacar que en todas las familias fueron únicamente las madres las que atendieron las visitas y participaron en las entrevistas, ausentándose la figura paterna por causas diversas.

Tanto las docentes como las familias participaron voluntariamente tras la explicación del propósito de la investigación por parte del equipo investigador al claustro del centro educativo, así como la descripción del mismo en las familias que fueron visitadas. Todos los participantes rellenaron un informe de consentimiento informado.

\section{Instrumentos de obtención de información}

Para obtener los resultados esperados se elaboraron diferentes instrumentos que pretendían recoger las respuestas de cada objetivo de investigación. Por un lado, para analizar la visión docente antes y después de la participación en el programa "fondos de conocimiento" se elaboró un cuestionario inicial y un post cuestionario, con preguntas de respuesta abierta. Por otro lado, con el fin de conocer la visión de las 6 familias participantes, se elaboró un guion que sirvió como instrumento para realizar las entrevistas semiestructuradas a las familias, posteriores a las visitas docentes en los hogares y a la participación de las familias en la escuela. A continuación, en el Cuadro 1, se detalla el contenido de los cuestionarios y entrevistas, describiendo las preguntas y su relación con los diferentes objetivos de la investigación: 
Cuadro 1

Contenido de los cuestionarios docentes y las entrevistas familiares

\begin{tabular}{lll}
\hline \multicolumn{1}{c}{ Cuestionario inicial } & \multicolumn{1}{c}{ Post cuestionario } & Entrevista semiestructurada \\
\hline $\begin{array}{l}\text { ¿Qué características definen para } \\
\text { ti una óptima relación familia- } \\
\text { escuela? (Objetivo 1) }\end{array}$ & $\begin{array}{l}\text { 1. Crees que la participación en el } \\
\text { programa ha tenido un impacto } \\
\text { en la relación de las familias y la } \\
\text { escuela? (Objetivo 3) }\end{array}$ & $\begin{array}{l}\text { ¿Qué significa para ti tener una } \\
\text { buena relación con la escuela? } \\
\text { (Objetivo 1) }\end{array}$ \\
$\begin{array}{l}\text { ¿Qué beneficios consideras que se } \\
\text { derivan de unas buenas relaciones } \\
\text { familia-escuela? (Objetivo 2) }\end{array}$ & $\begin{array}{l}\text { ¿Qué beneficios crees que se deri- } \\
\text { van de tener una buena relación } \\
\text { con la escuela? (Objetivo 2) }\end{array}$ \\
& $\begin{array}{l}\text { Ha habido algún cambio en vuestra } \\
\text { relación con la escuela después de } \\
\text { participar en el programa? } \\
\text { (Objetivo 3) }\end{array}$ \\
\hline
\end{tabular}

Nota. Entre paréntesis se indica el Objetivo de la investigación a la que la pregunta hace referencia.

Así pues, los datos empíricos se han recogido a partir de los cuestionarios, pre y post, así como de las entrevistas semiestructuradas en los hogares de las familias participantes.

\section{Procedimiento}

El proceso de aplicación del programa y recogida de datos tuvo una duración de un curso escolar. En una primera fase se elaboraron los instrumentos nombrados anteriormente: el pre y el post cuestionario y el guion de las entrevistas semiestructuradas, tomando como referencia los objetivos perseguidos en la investigación.

Fue durante la fase de formación del programa fondos de conocimiento al claustro educativo cuando se les pasó a las docentes participantes el cuestionario inicial por correo electrónico, y las respuestas fueron recibidas por la misma vía.

La segunda fase fue de asesoramiento a las docentes en la elaboración de las entrevistas a los hogares para detectar los fondos de conocimiento de las familias. En una tercera fase, se acompañó a las docentes en la detección de los fondos de conocimiento y en la posterior aplicación pedagógica en el aula, mediante las reuniones formadas por el grupo de estudio.

En una cuarta fase, posterior a la participación en el programa, nuestro objetivo era conocer qué impacto había causado. Para conocer el impacto en las docentes participantes, se les pasó el post cuestionario por correo electrónico y fue devuelto con las respuestas, de la misma manera que en el cuestionario inicial. Para valorar el impacto en las familias, la escuela nos facilitó el contacto de las mismas para acudir al hogar familiar y realizar las entrevistas semiestructuradas. Las entrevistas fueron grabadas - previo consentimiento de las familias- y posteriormente transcritas. También se utilizó un diario de campo para realizar anotaciones relevantes durante el periodo de recogida de datos y durante las reuniones con el grupo de estudio. Así pues, tras la aplicación de los instrumentos para la recogida de información, se analizaron los datos obtenidos siguiendo un procedimiento concreto, detallado a continuación.

\section{Análisis de datos}

Para realizar el análisis de los datos cualitativos, en un primer momento se realizó la transcripción de las grabaciones de las entrevistas a las familias y se recopilaron las respuestas de las docenes participantes.

Seguidamente, se utilizó el programa informático Atlas.ti versión 8.4.4 vinculado con la "teoría fundamenta" mencionada anteriormente. A partir de la incorporación de los cuestionarios y las entrevistas en el programa, se analizaron los datos mediante una codificación abierta, con el fin de resumir y agrupar conceptos relevantes en conceptos genéricos (categorización). Los conceptos genéricos (categorías principales) se asociaron a los 3 objetivos principales de este estudio:

- Conocer qué son unas óptimas relaciones familia-escuela para las docentes y las familias participantes; 
- Identificar los beneficios que se derivan de unas óptimas relaciones familia-escuela según docentes y familias participantes $y$, finalmente;

- Analizar el impacto del programa en la mejora de las relaciones familia-escuela según docentes y familias participantes.

De estos conceptos genéricos - categorías principales- se realizó una subcategorización en base a las respuestas obtenidas por parte de docentes y familias. Con el objetivo de facilitar la presentación de dicho análisis, y los resultados hallados, se han creado 3 cuadros comparativos, uno por cada categoría de análisis, con las respuestas de las docentes y de las familias y con el número de veces que aparece citada cada respuesta (Cuadros 2,3 y 4 ).

\section{Resultados}

Los resultados se dividen en las tres grandes categorías, que se relacionan directamente con los tres objetivos principales de esta investigación (mencionados anteriormente). En primer lugar, se analizan las respuestas de familias y docentes en relación a la percepción sobre lo que son unas "óptimas relaciones familia-escuela". En segundo lugar, sobre las creencias de los "beneficios que se derivan de unas óptimas relaciones familia-escuela" desde la óptica docente y de las familias. Por último, se analiza el "impacto del programa en la relación familia-escuela" según las docentes y las familias participantes en el programa. Las citas asociadas a dichas categorías son el resultado del proceso de codificación a partir de la presencia de informaciones vinculadas a las mismas en las distintas entrevistas realizadas. De manera que un mismo tema pudiera aparecer más de 6 veces, familias participantes, al aparecer en distintos momentos de la entrevista transcrita.

Las citas asociadas a dichas categorías son el resultado del proceso de codificación a partir de la presencia de informaciones obtenidas mediante los instrumentos de recogida de datos utilizados. En el caso de las docentes, al responder a preguntas cerradas de un cuestionario, el número de citas se corresponde con el número de docentes que han dado cada respuesta. En el caso de las familias, al aparecer un mismo tema en distintos momentos de la entrevista transcrita, se ha especificado en cada caso el número de veces citada y, además, el número de familias que cita cada categoría para valorar de manera más precisa la relevancia de dicha categoría.

\subsection{Definición de unas óptimas relaciones familia-escuela}

En total se han identificado 8 aspectos (4 según docentes y 4 según familias) que definen unas óptimas relaciones familia-escuela (Cuadro 2).

Cuadro 2

Definición de unas óptimas relaciones familia-escuela según docentes y familias

\begin{tabular}{|c|c|c|c|c|}
\hline \multicolumn{2}{|c|}{ Según docentes } & \multicolumn{3}{|c|}{ Según familias } \\
\hline Categoria & $\begin{array}{l}\text { Número de } \\
\text { veces citada }\end{array}$ & Categoria & $\begin{array}{l}\text { Número de veces } \\
\text { citada }\end{array}$ & $\begin{array}{c}\text { Número de } \\
\text { familias que lo } \\
\text { citan }\end{array}$ \\
\hline $\begin{array}{l}\text { Participación de las fami- } \\
\text { lias en las tareas escolares }\end{array}$ & 6 & $\begin{array}{l}\text { Compartir espacios entre } \\
\text { docentes y familias }\end{array}$ & 9 & 5 \\
\hline $\begin{array}{l}\text { Comunicación entre la } \\
\text { familia y la escuela }\end{array}$ & 6 & $\begin{array}{l}\text { Comunicación entre la } \\
\text { familia y la escuela }\end{array}$ & 7 & 4 \\
\hline $\begin{array}{l}\text { Vínculo de confianza } \\
\text { mutua }\end{array}$ & 6 & $\begin{array}{l}\text { Presencia de las diferentes } \\
\text { culturas en la escuela }\end{array}$ & 4 & 3 \\
\hline $\begin{array}{l}\text { Responsabilidad compar- } \\
\text { tida }\end{array}$ & 2 & $\begin{array}{l}\text { Vínculo de confianza } \\
\text { mutua }\end{array}$ & 3 & 3 \\
\hline
\end{tabular}


Las docentes ponen énfasis en la participación de las familias en las tareas escolares "las familias tienen que mostrar interés y participación en las actividades y aprendizajes que realicen sus hijos e hijas”, en la comunicación entre la familia "desde la escuela también debemos informar a las familias de las actividades y aprendizajes que realicen sus hijos/as" / "dar la información necesaria ambas partes con el fin de favorecer el progreso y crecimiento del niño". También resaltan la necesidad de crear un vínculo de confianza mutua "pienso que son muy importantes los factores acogida y confianza entre las dos partes" y la responsabilidad compartida entre familia y escuela "las buenas relaciones deberían ser de trabajo compartido y responsabilidad compartida. No culparse sino ir cogidos de la mano y estar de acuerdo con las propuestas que se van haciendo por las dos partes".

Las familias acentúan, principalmente (en cinco de las seis familias participantes), la necesidad de compartir espacios entre docentes y familias "puede ser una excursión o algo así" / "Preguntar. Cómo en entrevista, ¿o este no? Venir más a casa. Conocernos más también fuera de la escuela”, así como también la comunicación "claro, es importante para los padres que conozcan qué hace el niño en clase" / "Sí, aunque tendríamos que hablar más". También resaltan, tres de las seis familias participantes, la importancia de dar presencia a las diferentes culturas en la escuela "Y un día yo le he dicho a una profesora: ¿por qué no hacemos una placa en la entrada de bienvenidos con muchos idiomas? Porque hay muchos en la escuela. Hay africanos, hay de India me parece, Pakistán... Y hacemos todos los idiomas. Y me ha dicho que un día trabajaremos en esto". Por último, coincidiendo con la opinión docente, consideran relevante, también tres de las seis familias participantes, la creación de un vínculo de confianza mutua "yo desde siempre he tenido muy buena conexión con la maestra. Esto es muy importante".

\subsection{Beneficios de unas buenas relaciones familia-escuela}

Respecto a los beneficios que se derivan de unas buenas relaciones familia-escuela (véase Cuadro 3), se han categorizado un total de 11 (5 según docentes y 6 según familias).

Cuadro 3

Beneficios de unas buenas relaciones familia-escuela según docentes y familias

\begin{tabular}{|c|c|c|c|c|}
\hline \multicolumn{2}{|l|}{ Según docentes } & \multicolumn{3}{|c|}{ Según familias } \\
\hline Categoria & $\begin{array}{l}\text { Número } \\
\text { de veces } \\
\text { citada }\end{array}$ & Categoria & $\begin{array}{l}\text { Número de } \\
\text { veces citada }\end{array}$ & $\begin{array}{l}\text { Número de } \\
\text { familias que lo } \\
\text { citan }\end{array}$ \\
\hline $\begin{array}{l}\text { Todos se sienten parte de un } \\
\text { todo }\end{array}$ & 2 & $\begin{array}{l}\text { Los niños y niñas van más } \\
\text { contentos a la escuela }\end{array}$ & 7 & 6 \\
\hline Participación más agradable & 2 & $\begin{array}{l}\text { Los niños y niñas estable- } \\
\text { cen un vínculo más fuerte } \\
\text { con la maestra. }\end{array}$ & 2 & 2 \\
\hline Confianza mutua & 2 & Confianza mutua & 5 & 4 \\
\hline Objetivos educativos comunes & 5 & $\begin{array}{l}\text { Objetivos educativos } \\
\text { comunes }\end{array}$ & 4 & 4 \\
\hline \multirow{2}{*}{$\begin{array}{l}\text { Clima de aprendizaje más } \\
\text { favorable para el alumno }\end{array}$} & & $\begin{array}{l}\text { Conocimiento y respeto } \\
\text { por las diferentes culturas. }\end{array}$ & 8 & 4 \\
\hline & 4 & $\begin{array}{l}\text { Refuerzo en casa del traba- } \\
\text { jo escolar }\end{array}$ & 1 & 1 \\
\hline
\end{tabular}

Las docentes consideran que una buena relación entre familia y escuela favorece que todos los integrantes se sientan parte de la entidad escolar "que todo el mundo: hijos, alumnos, maestros y familias se sienten parte de un todo, de algo más grande", que su participación en la escuela sea más agradable "el hecho de tener una buena relación familia-escuela hará más agradable la organización de actividades, la colaboración y la participación". Hablan también de la necesidad de generar una confianza mutua "en definitiva, lo más importante es la confianza", también de que se establezcan objetivos educativos comunes "el niño/a recibe siempre el mismo mensaje, tanto en la escuela como en casa, hecho que reafirma la tarea 
escolar y la de la familia", y que se genere un clima de aprendizaje más favorable para el alumnado "una buena relación favorecerá que el alumno aprenda en un mejor clima de trabajo".

Por otra parte, las familias, casi en su totalidad (cinco de las seis participantes), consideran que sus hijos/ as van más contentos a la escuela "los niños van al cole contentos", que se genera un clima de confianza mutua entre familias y escuela "cuando hagamos cosas juntos, nosotros sabemos de ellos y ellos también saben de nosotros. Esto para mí es muy importante"; también remarcan la necesidad de conocer y, por tanto, de respetar las diferentes culturas que conviven en la escuela "Porque así ellos conocen un poquito de nosotros, de nuestra cultura. Porque también creo que es importante saber cómo vamos en nuestro país y conocer un poco más”. La visión en común entre familias y docentes respecto a los beneficios que se derivan es que se establecen objetivos educativos comunes, lo que permite conducir la educación de sus hijos/as hacia una misma dirección "Sí, es muy importante. Para mi es muy importante ver que entre las dos vamos al mismo sitio, sabes?"| "nos unimos porque tenemos el mismo objetivo". Por otra parte, dos de las familias participantes se refieren al establecimiento de un vínculo más fuerte con la maestra: "porque, bueno, porque los niños ven esto y tienen más confianza con las maestras"; y una de las familias se refiere a la posibilidad de reforzar en casa el trabajo escolar "en casa, buscar como poder reforzar lo que se hace en la escuela, pero eso también viene dado por la conexión con la escuela".

\subsection{Impacto del programa en la mejora de las relaciones familia-escuela}

Por lo que se refiere al impacto que ha generado la aplicación del programa fondos de conocimiento en la mejora de las relaciones familia-escuela (ver cuadro 4), se han categorizado un total de 13 respuestas (7 por parte de las docentes, 6 por parte de las familias).

Cuadro 4

Impacto del programa en la mejora de las relaciones familia-escuela según docentes y familias

\begin{tabular}{|c|c|c|c|c|}
\hline \multicolumn{2}{|c|}{ Según docentes } & \multicolumn{3}{|c|}{ Según familias } \\
\hline Categoria & $\begin{array}{l}\text { Número de veces } \\
\text { citada }\end{array}$ & Categoria & $\begin{array}{l}\text { Número de } \\
\text { veces citada }\end{array}$ & $\begin{array}{c}\text { Número } \\
\text { de familias } \\
\text { que lo citan }\end{array}$ \\
\hline $\begin{array}{l}\text { Las familias participan más de } \\
\text { la vida en el aula }\end{array}$ & 2 & $\begin{array}{l}\text { Más comunicación con } \\
\text { la maestra }\end{array}$ & 3 & 3 \\
\hline $\begin{array}{l}\text { Relación más cercana entre la } \\
\text { familia y la escuela }\end{array}$ & 6 & \multirow{2}{*}{$\begin{array}{l}\text { Mayor confianza con la } \\
\text { maestra }\end{array}$} & \multirow{2}{*}{7} & \multirow{2}{*}{5} \\
\hline $\begin{array}{l}\text { Familias más comunicativas } \\
\text { con la maestra }\end{array}$ & 3 & & & \\
\hline $\begin{array}{l}\text { Se generan espacios para } \\
\text { compartir experiencias y } \\
\text { anécdotas }\end{array}$ & 1 & $\begin{array}{l}\text { Los niños y niñas se } \\
\text { muestran más cerca- } \\
\text { nos con la maestra }\end{array}$ & 3 & 3 \\
\hline $\begin{array}{l}\text { Mayor conocimiento de la rea- } \\
\text { lidad y las necesidades de cada } \\
\text { familia }\end{array}$ & 2 & $\begin{array}{l}\text { Mayor participación en } \\
\text { la escuela. }\end{array}$ & 5 & 4 \\
\hline $\begin{array}{l}\text { Conocimiento del núcleo } \\
\text { familiar, rutinas y tradiciones }\end{array}$ & 2 & $\begin{array}{l}\text { Incorporación de nue- } \\
\text { vas rutinas en el hogar } \\
\text { familiar. }\end{array}$ & 1 & 1 \\
\hline $\begin{array}{l}\text { Las familias se sienten } \\
\text { valoradas }\end{array}$ & 1 & $\begin{array}{l}\text { Valorización de los } \\
\text { conocimientos de la } \\
\text { familia }\end{array}$ & 8 & 6 \\
\hline
\end{tabular}

Las docentes han observado que las familias participan más de la vida en el aula "las familias se han mostrado más cercanas y participativas de la vida en el aula" / "las familias que han participado han mostrado más interés y ganas de participar", que existe una relación más cercana entre la familia y la escuela "ha mejorado mucho la relación, ya que nos ha permitido establecer relaciones más cercanas con las familias" / "el hecho de poder ir a casa y hablar en un momento relajado y tranquilo ha mejorado la complicidad 
de la relación". También destacan que las familias se muestran más comunicativas con la maestra "las vemos más cercanas a la hora de comunicarse con nosotras" / "Ellas mismas o a través de sus hijos e hijas intentan preguntar cuando no entienden y muestran más iniciativa para pedirnos explicaciones". También resaltan que las entrevistas han permitido generar más espacios para compartir experiencias y anécdotas "compartir experiencias y anécdotas en un contexto relajado" ..., así como también les han permitido conocer mejor la realidad y necesidades de cada familia "ahora entendemos mejor cuál es la realidad que tienen en casa" / "nos ayuda mucho al nivel de saber qué necesidades tienen como familia". También, visitar el hogar familiar les ha permitido conocer mejor el núcleo familiar, las rutinas y las tradiciones "conocemos la familia y la vemos desde otra óptica, otra perspectiva" / "Nos permite conocer el núcleo familiar, las dinámicas y las tradiciones de cada familia”. Por último, destacan que las familias se han sentido valoradas "se han sentido valoradas al venir a la escuela a enseñar los conocimientos que tienen y eso ha mejorado su autoconcepto".

A su vez, las familias sienten que ahora tienen más comunicación con la maestra, así lo afirman tres de las familias participantes: "sí, porque fuera solo "hola", le dejo al niño y vuelvo. Casi no quedamos nada, pero aquí seguro hablamos más y también más confianza" / "yo he conocido más a la profesora y tengo más confianza con ella. Y me da más confianza la escuela, porque como es una escuela de muchas culturas tenía mucha desconfianza, la verdad” / "Sí, sí, después de las visitas tenemos más confianza. Sí porque así ellas ya saben más sobre nosotros. Porque, bueno, siempre he dicho que son muy buenas. Me encantan como maestras". Cinco de las familias entrevistadas destacan, también, que sus hijos/as se muestran más cercanos con la maestra "aquí está muy contenta. Me dice que ha venido la maestra, que estaba muy contenta, de verdad". Una familia resalta que, posteriormente a la visita, ha incorporado nuevas rutinas en el hogar "desde que vino a casa siempre leemos un cuento a la noche, una idea que antes no hacíamos". En común con las docentes, cuatro de las familias visitadas aseguran que les ha permitido tener una mayor participación en la escuela" Bien. Algo diferente. Ya te he dicho, desde que comienza mi hija mayor aquí, desde ahí nunca lo he hecho, ahora está en sexto, seis años nunca... Y me gusta. No tengo ningún problema mientras que yo no trabajo". Sin embargo, la categoría que se observa en todas las familias participantes se refiere, de alguna manera, a su sentimiento de valoración y reconocimiento resultado de la visita por parte de las docentes: "me gusta que las maestras también participaban y se interesaban en aprender cosas nuevas" / "Puedo hacer alguna cosa aquí que les puede gustar" / "Sí, sí. Yo he sabido hacer cosas que aquí no sabían. Yo estaba contenta por esto”.

\section{Discusión y conclusiones}

El objetivo general de la investigación fue identificar los factores que inciden en la mejora de las relaciones familia-escuela, tanto desde la visión de las docentes como de las familias participantes en el estudio. En este caso, por primera vez en la literatura sobre el impacto de la aproximación de los fondos de conocimiento (Esteban-Guitart y Vila, 2013a; Lin y Bates, 2010; Llopart et al., 2018; Meyer y Mann, 2006; Tenery, 2005; Whyte y Karabon, 2016), se ha incorporado en el análisis la voz de las familias.

Los resultados muestran que las docentes, antes de la aplicación del programa, consideraron que unas óptimas relaciones familia-escuela se basan en la participación de las familias en las tareas escolares, en la comunicación entre la escuela y la familia, en un mayor vínculo de confianza mutua y en la responsabilidad compartida. Estas respuestas podrían vincularse con la perspectiva del déficit (Valencia, 2010) en el sentido de aludir a determinadas insuficiencias de las familias, como por ejemplo su pobre participación en las tareas escolares. El estudio de Collet y otros (2014) concluía que la visión docente respecto a las familias se basaba en la falta de implicación, seguimiento e interés en lo escolar. En contraposición, se supone que debe ser la escuela, como institución pública de acogida y fomento de la igualdad y la cohesión social, quién incorpore en su ejercicio la tarea de aproximarse respetuosamente a las familias, ofreciéndoles espacios para su reconocimiento y legitimación (Collet et al., 2014; Poveda, 2001). En este sentido, además de coincidir con la importancia de la comunicación y la vinculación, cinco de las familias también resaltaron principalmente la necesidad de compartir espacios con las docentes, así como la importancia de la presencia de las diferentes culturas en la escuela, por parte de tres de las seis familias participantes. Resultados que refuerzan la idea de concebir la escuela como espacio democrático para el intercambio, 
reconocimiento y sostenibilidad de las distintas formas lingüísticas, identitarias y culturales que conviven en un mismo territorio (Esteban-Guitart et al., 2019).

En relación a los beneficios de unas buenas relaciones familia-escuela, las docentes y cuatro de las familias coinciden en la importancia de la confianza mutua y el establecimiento de objetivos educativos comunes. Lo que se relaciona con los elementos considerados "esenciales" de la aproximación de los fondos de conocimiento. En particular, el establecimiento de relaciones basadas en la confianza mutua por parte de docentes y familias a partir de la discusión sobre las prácticas, destrezas y recursos que éstas poseen (Moll, 2019). Sin embargo, en relación a los "objetivos educativos comunes", considerados en el estudio aquí descrito tanto por parte de las docentes como en cuatro de las familias participantes, supone una reconsideración del marco original de la aproximación de los fondos de conocimiento. Específicamente, se supone la creación de un "grupo de estudio" entre docentes e investigadores de la universidad, considerado como una "comunidad de práctica", contexto de formación y re-actualización docente (Esteban-Guitart et al., 2018), que permite a los y las docentes formarse tanto teórica, metodológica como analíticamente para llevar a cabo las visitas, la identificación de los fondos de conocimiento, y su vinculación con el currículum y objetivos pedagógicos (González et al., 2005). Sin embargo, la participación de las familias en dichos grupos de estudio es inédita, lo que sugiere que futuras aplicaciones deberían contemplar una mayor participación, no tanto en las actividades de aula (ver, por ejemplo, Jovés et al., 2015), sino también en el grupo de estudio propiamente dicho; aspecto que facilitaría la creación de objetivos educativos comunes, así como una mayor colaboración e intercambio. Destaca, en todas las familias entrevistadas, la percepción según la cual todos los niños y niñas van más contentos a la escuela. Lo que se podría vincular con los resultados de dos recientes investigaciones que documenta los beneficios de la aproximación de los fondos de conocimiento en el bienestar y clima en el aula (Flint y Jaggers, 2021; Volman y Gilde, 2021).

Por último, se han documentado una serie de beneficios derivados de la aplicación de la aproximación de los fondos de conocimiento que van en la misma línea que la literatura previa existente al respecto (Esteban-Guitart y Vila, 2013a; Flint y Jaggers, 2021; Lin y Bates, 2010; Llopart et al., 2018; Meyer y Mann, 2006; Tenery, 2005; Volman y Gilde, 2021; Whyte y Karabon, 2016). En general, se concluye que, según la percepción de las docentes participantes, han mejorado las relaciones con las familias, siendo más cercanas, con una mayor participación de las familias en el aula. También se valora el mayor conocimiento que se obtiene del núcleo familiar, sus rutinas, saberes y destrezas, así como de las circunstancias y necesidades del alumnado y familias visitadas. Un conocimiento y relación más estrecha que se vincula a una mayor comprensión de las situaciones de cada alumno y alumna, así como un desmantelamiento de prejuicios y estereotipos a partir del conocimiento real de la vida de los estudiantes y sus familias (Esteban-Guitart y Vila, 2013a; Lin y Bates, 2010; Meyer y Mann, 2006). Por parte de las familias, destaca la categoría "mayor confianza con la maestra", seguida de una mayor participación en la escuela, una mejor comunicación con la maestra, un acercamiento del alumnado hacia la maestra y contexto educativo escolar y, especialmente, se valora la puesta en consideración de las destrezas y saberes de las familias. En conclusión, las familias enfatizan como beneficios de la aproximación una valorización de sus conocimientos y habilidades, así como una mayor confianza con las docentes.

Sin embargo, dichos resultados deben de tomarse en cautela como mínimo debido a tres razones. En primer lugar, el carácter cualitativo de la investigación imposibilita la generalización de los resultados obtenidos. En segundo lugar, las percepciones de las familias se obtuvieron, solamente, a partir de una entrevista a las mismas; lo que constituye una limitación del estudio realizado. En tercer lugar, no se ha implementado un grupo control que hubiera permitido contrastar los resultados hallados. Futuras investigaciones, en este sentido, deberían documentar con una mayor profundidad los procesos de cambio en las relaciones familia-escuela tanto antes, como después, de recibir las visitas docentes; así como a través de un grupo control formado por docentes y familias no participantes en el programa fondos de conocimiento. Ello permitiría identificar la existencia de factores exógenos a la implementación del programa que pudieran influir en las visiones y respuestas obtenidos. Especialmente relevante fuera para contribuir al objetivo específico tres de la investigación vinculado a la identificación de la contribución del programa en la mejora de las relaciones familia-escuela según docentes y familias participantes. Por otra parte, solamente se ha documentado la opinión y valoración de las familias tras las visitas de las docentes, no anteriormente a la aplicación de los fondos de conocimiento, lo que enriquecería la valoración del impacto del programa. 
En conclusión, se han presentado datos que refuerzan los beneficios de las relaciones familia-escuela según la percepción de las mismas docentes, así como, por primera vez en la literatura disponible, el impacto de la aproximación de los fondos de conocimiento por parte de las familias visitadas. Sugerimos, en este sentido, la necesidad de incorporar sus voces no solamente en la identificación de los fondos de conocimiento, lo que se sugiere en la aproximación original (González et al., 2005; Moll et al., 1992), sino también en los "grupos de estudio" formados por docentes e investigadores e investigadoras de la universidad. Ello supondría un enriquecimiento de la aproximación, así como una manera de avanzar en uno de los objetivos nucleares de los fondos de conocimiento, en particular, y en la mejora de las relaciones familia-escuela, en general, al saber: el establecimiento de relaciones basadas en la colaboración, intercambio y confianza mutua.

\section{Referencias}

Ainscow, M. (2012). Haciendo que las escuelas sean más inclusivas: Lecciones a partir del análisis de la investigación internacional. Revista de Educación Inclusiva, 5(1), 39-49.

Besalú, X. y Vila, I. (2007). La buena educación. Libertad e igualdad en la escuela del siglo XXI. Los Libros de la Catarata.

Blasco, J. (2018). Què funciona en els programes de foment de la implicació parental? Fundació Bofill.

Bronfenbrenner, U. (1979). La ecología del desarrollo humano. Paidós.

Collet, J., Besalú, X., Feu, J. y Tort, A. (2014). Escuelas, familias y resultados académicos. Un nuevo modelo de análisis de las relaciones entre docentes y progenitores para el éxito de todo el alumnado. Profesorado, Revista de currículum y formación del profesorado, 18(2) 7-33.

Crosnoe, R. (2012). Family-school connections, early learning and socioeconomic inequality in the US. Multidisciplinary Journal of Educational Research, 2(1), 1-36. https://doi.org/10.4471/remie.2012.0

Epstein, J. L. (2018). School, family and community partnerships: Preparing educators and improving schools. Routledge.

Esteban-Guitart, M. (2021). Advancing the funds of identity theory: A critical and unfinished dialogue. Mind, Culture and Activity. https://doi.org/10.1080/10749039.2021.1913751

Esteban-Guitart, M. y Bastiani, J. (2010). ¿Puede un modelo educativo intercultural combatir la discriminación y la xenofobia? Athenea Digital: Revista de Pensamiento e Investigación Social, 17, 3-16.

https://doi.org/10.5565/rev/athenead/v0n17.500

Esteban-Guitart, M. y Saubich, X. (2013). La práctica educativa desde la perspectiva de los fondos de conocimiento e identidad. Teoría de la Educación: Revista Interuniversitaria, 25(2), 189-211.

Esteban-Guitart, M. y Vila, I. (2013a). La modificación de las creencias sobre las familias de origen inmigrante por parte de docentes después de participar en el proyecto educativo "fondos de conocimiento". Cultura y Educación, 25(2), 241-254. https://doi.org/10.1174/113564013806631282

Esteban-Guitart, M. y Vila, I. (2013b). Experiencias en educación inclusiva. Vinculación escuela, familia y comunidad. Horsori.

Esteban-Guitart, M. y Moll, L. (2014a). Funds of identity: A new concept based on funds of knowledge approach. Culture and Psychology, 20(1), 31-48. https://doi.org/10.1177/1354067X13515934

Esteban-Guitart, M. y Moll, L. (2014b). Lived experiences, funds of identity and education. Culture and Psychology, 20(1), 70-81. https://doi.org/10.1177/1354067X13515940

Esteban-Guitart, M., Serra,J. M. y Llopart, M. (2018). The role of the study group in the funds of knowledge approach. Mind, Culture, and Activity, 25(3), 216-228. https://doi.org/10.1080/10749039.2018.1448871

Esteban-Guitart, M., Lalueza, J. L., Zhang-Yu, C. y Llopart, M. (2019). Sustaining students' cultures and identities. A qualitative study based on the funds of knowledge and identity approaches. Sustainability, 11(12), 34-47. https://doi.org/10.3390/su11123400

Essomba, M. (2003). Educación e inclusión social de inmigrados y minorías. Tejer redes de sentido compartido. Praxis. 
Flint, A. S. y Jaggers, W. (2021). You matter here: The impact of asset-based pedagogies on learning. Theory into Practice. https://doi.org/10.1080/00405841.2021.1911483

García-Bacete, F. J. (2003). Las relaciones escuela-familia: Un reto educativo. Infancia y Aprendizaje, 26(4), 425 - 437. https://doi.org/10.1174/021037003322553824

Gifre, M. y Esteban-Guitart, M. (2012). Consideraciones educativas de la perspectiva ecológica de Urie Bronfenbrenner. Contextos Educativos. Revista de Educación, 15, 79-92.

Glaser, B. G. y Strauss, A. (1967). Discovery of grounded theory: Strategies for qualitative research. Sociology Press.

González, N. y Moll, L. C. (2002). Cruzando el puente: Building bridges to funds of knowledge. Educational Policy, 16(4), 623-641. https://doi.org/10.1177/0895904802016004009

González, N., Moll, L. C. y Amanti, C. (2005). Funds of knowledge: Theorizing practices in households, communities, and classrooms. Routledge.

Henderson, A. T. y Mapp, K. L. (2002). A new wave of evidence. The impact of school, family and community connections on student achievement. National Center for family \& Community Connections with Schools.

Hernández, R. M. (2014). La investigación cualitativa a través de entrevistas: su análisis mediante la teoría fundamentada. Cuestiones Pedagógicas, 23, 187-210. https://doi.org/10.12795/CP

Hogg, L. (2011). Funds of knowledge: An investigation of coherence within the literature. Teaching and Teacher Education, 27(3), 666-677. https://doi.org/10.1016/j.tate.2010.11.005

Jovés, P., Siqués, C. y Esteban-Guitart, M. (2015). The incorporation of funds of knowledge and funds of identity of students and their families into educational practice: A case study from Catalonia, Spain. Teaching and Teacher Education, 49, 68-77. https://doi.org/10.1016/j.tate.2015.03.001

Lin, M. y Bates, B. (2010). Home visits: How do they affect teachers' beliefs about teaching and diversity? Early Childhood Education Journal, 38(3), 179-185. https://doi.org/10.1007/s10643-010-0393-1

Llopart, M. y Esteban-Guitart, M. (2018). Funds of knowledge in 21st century societies: Inclusive educational practices for under-represented students. A literature review. Journal of Curriculum Studies, 50(2), 145-161. https://doi.org/10.1080/00220272.2016.1247913

Llopart, M., Vilagran, I., Güell, C. y Esteban-Guitart, M. (2017). Las visitas etnográficas a los hogares de los estudiantes como estrategia para crear lazos de confianza entre docentes y familias. Pedagogia $i$ Treball Social, 6(1), 70-95.

Llopart, M., Serra, J. M. y Esteban-Guitart, M. (2018). Teachers' perceptions of the benefits, limitations, and areas for improvement of the funds of knowledge approach: A qualitative study. Teachers and Teaching, 24(5), 571-583. https://doi.org/10.1080/13540602.2018.1452729

Meyer, J. A. y Mann, M. B. (2006). Teachers' perceptions of the benefits of home visits for early elementary children. Early Childhood Education Journal, 34(1), 93-97. https://doi.org/10.1007/s10643-006-0113-z

Moll, L. (2019). Elaborating funds of knowledge: Community-oriented practices in international contexts. Literacy Research: Theory, Method and Practice, 68(1), 130-138. https://doi.org/10.1177/2381336919870805

Moll, L., Amanti, C., Neff, D. y González, N. (1992). Funds of knowledge for teaching: Using a qualitative approach to connect homes and classrooms. Theory into Practice, 31(2),132-141. https://doi.org/10.1080/00405849209543534

Poveda, D. (2001). La educación de las minorías étnicas desde el marco de las continuidades-discontinuidades familia-escuela. Gazeta de Antropología, 17, 1-15.

Rodríguez-Ruiz, B., Álvarez-Blanco, L., Martínez-González, R. y Epstein, J. L. (2019). Presentación del número Relación entre centros educativos, familias y entidades comunitarias. Aula Abierta, 48(1), 7-10.

Semke, C. A. y Sheridan, S. M. (2012). Family-school connections in rural educational settings: A systematic review of the empirical literature. School Community Journal, 22(1), 21-48.

Sharma, M. (2018). Seeping deficit thinking assumptions maintain the neoliberal education agenda: Exploring three conceptual frameworks of deficit thinking in inner-city schools. Education and Urban Society, 50(2), 136 154. https://doi.org/10.1177/0013124516682301 
Tenery, M. F. (2005). La visita. In N. González, L. C. Moll y C. Amanti (Eds.), Funds of knowledge: Theorizing practices in households, communities and classrooms (pp. 119-130). Lawrence Erlbaum Associates.

Valencia, R. R. (2010). Dismantling contemporary deficit thinking: Educational thought and practice. Routledge.

Volman, M. y Gilde, J. (2021). The effects of using students' funds of knowledge on educational outcomes in the social and personal domain. Learning, Culture and Social Interaction, 28, 100472.

https://doi.org/10.1016/j.1csi.2020.100472

Vila, I. y Esteban-Guitart, M. (2017). Familia, escuela y comunidad en las sociedades del siglo XXI. Horsori.

Whyte, K. y Karabon, A. (2016). Transforming teacher-family relationships: Shifting roles and perceptions of home visits through the funds of knowledge approach. Early Years. An International Journal, 36(2), 207-221.

https://doi.org/10.1080/09575146.2016.1139546

\section{Breve CV de la autora}

\section{Mireia Machancoses}

Graduada en Maestra de Educación Primaria en la Universidad de Valencia. Máster en Atención a la Diversidad en una Educación Inclusiva en la Universidad de Girona. Estudiante del Doctorado Interuniversitario en Psicología de la Educación (DIPE) en la Universidad de Girona, dentro del grupo de investigación “Cultura y Educación”. Desarrollando la tesis doctoral sobre el impacto de la aproximación de los fondos de conocimiento en las relaciones familia-escuela en contextos marcados por una elevada diversidad social y cultural. Maestra en un Centro Educativo de Educación Especial con alumnado con diversidad funcional neuromotora. Email: mireiareal@gmail.com

ORCID ID: https://orcid.org/0000-0003-4932-5386 